\title{
A model for the dynamics of gas bubbles in soft tissue
}

\author{
Xinmai Yang a) and Charles C. Church \\ National Center for Physical Acoustics, The University of Mississippi, University, Mississippi 38677
}

(Received 21 April 2005; revised 16 September 2005; accepted 17 September 2005)

\begin{abstract}
Understanding the behavior of cavitation bubbles driven by ultrasonic fields is an important problem in biomedical acoustics. Keller-Miksis equation, which can account for the large amplitude oscillations of bubbles, is rederived in this paper and combined with a viscoelastic model to account for the strain-stress relation. The viscoelastic model used in this study is the Voigt model. It is shown that only the viscous damping term in the original equation needs to be modified to account for the effect of elasticity. With experiment determined viscoelastic properties, the effects of elasticity on bubble oscillations are studied. Specifically, the inertial cavitation thresholds are determined using $R_{\max } / R_{0}$, and subharmonic signals from the emission of an oscillating bubble are estimated. The results show that the presence of the elasticity increases the threshold pressure for a bubble to oscillate inertially, and subharmonic signals may only be detectable in certain ranges of radius and pressure amplitude. These results should be easy to verify experimentally, and they may also be useful in cavitation detection and bubble-enhanced imaging. (C) 2005 Acoustical Society of America. [DOI: 10.1121/1.2118307]
\end{abstract}

PACS number(s): 43.35.Wa, 43.80.Sh, 43.35.Ei [FD]

Pages: $3595-3606$

\section{INTRODUCTION}

Cavitation phenomena are very complicated due to the nonlinear oscillations of small bubbles and the interactions between these bubbles. In most cases, cavitation occurs in water, which is the most familiar fluid to us, and as a result, studies of bubble dynamics in water have been undertaken for over 80 years. Bubble dynamic models are well established for bubbles in water or simple Newtonian fluids. With the development of new materials and new techniques, the study of bubble dynamics in viscoelastic media becomes necessary. The increasing interest in cavitation is partly related to the application of medical ultrasound. For example, the use of bubble-based contrast agents in diagnostic ultrasound has significantly increased the quality of imaging. Recently, this issue has become more important due to the development of the high intensity focused ultrasound (HIFU) for therapeutic medicine. High intensity ultrasound will induce cavitation in soft tissue, and these microbubbles have a huge impact on the distribution of the ultrasound energy. In these situations, the surrounding media, i.e., biological tissues, often exhibit non-Newtonian behavior. Understanding the behavior of cavitation in vivo may provide a powerful tool to improve the quality of medical ultrasound.

The study of these microbubbles involves bubble oscillations in viscoelastic media. Many researchers have extended the analysis of bubble dynamics in Newtonian fluids to non-Newtonian fluids. Fogler and Goddard ${ }^{1}$ combined the linear Maxwell model with the Rayleigh-Plesset equation and examined the collapse of a spherical cavity in a large body of an incompressible viscoelastic liquid. The bubble was modeled as a void, and the effect of elasticity was investigated. Their results showed that the elasticity in the liquid can significantly retard the collapse of a bubble. A three

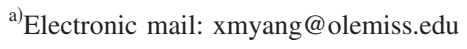

parameter linear Oldroyd model was employed by Tanasawa and Yang $^{2}$ to study the free oscillation of a gas bubble in viscoelastic fluids. They investigated the effects of the viscous damping in the presence of elasticity, and found that in the presence of elasticity, the effect of viscous damping on bubble collapse is less than that in the pure fluid. Later, Shima, Tsujina, and Nanjo ${ }^{3}$ investigated the nonlinear oscillations of gas bubbles in viscoelastic fluids using the model first derived by Tanasawa and Yang, ${ }^{2}$ and the effects of relaxation time and retardation time were clarified. A fully numerical scheme was developed by $\mathrm{Kim}^{4}$ to investigate collapse of a spherical bubble in a large body of UpperConvective Maxwell fluid. He observed that fluid elasticity accelerated the collapse in the early stage of collapse while in the later stages it retarded the collapse. His approach was very computationally intensive. Alekseev and Rybak ${ }^{5}$ presented the resonance frequency of gas bubbles in elastic media. The dispersion equation in a viscoelastic medium was also derived for bubble clouds in their study. Allen and Roy ${ }^{6}$ chose the linear Maxwell and Jeffreys models as the liquid constitutive equation to study bubble oscillations in linear viscoelastic fluids. After linearization of the original nonlinear differential equation (a Rayleigh-Plesset type equation), analytical solutions were obtained and compared with the Newtonian results. In a later study of nonlinear viscoelasticity, they ${ }^{7}$ employed the Upper-Convective Maxwell model as the constitutive equation with the Rayleigh-Plesset equation. A fully numerical study was conducted to solve the governing system of equations. The results of the linear and nonlinear viscoelastic approaches were compared. Their results showed that tissue viscoelasticity may be important for the potential cavitation bioeffects.

For diagnostic ultrasound examinations, the acoustic intensity is usually insufficient to induce inertial cavitation in soft tissue directly. ${ }^{8}$ The only source of microbubbles in soft tissue would be direct injection of a bubble-based contrast 
agent, an uncommon procedure. However, the use of higher intensity ultrasound, e.g., HIFU therapy, will cause cavitation in soft tissue directly. Different from contrast agent bubbles, this cavitation involves free bubbles oscillating nonlinearly because of the high intensity of the sound field. Previous models for viscoelastic media are all based on the RayleighPlesset equation, which is not very appropriate for large amplitude oscillations. In this work, we seek a model that is capable of accounting for the potentially large-amplitude oscillations of bubbles exposed to HIFU fields. In addition, the bubble model must incorporate a viscoelastic model consistent with measured tissue properties. Although the data on viscoelastic properties of soft tissue at megahertz frequencies are very limited, the linear Voigt model has proven appropriate for the tissues studied; ${ }^{9,10}$ none of the above-mentioned models incorporates these experimental results. The KellerMiksis equation ${ }^{11}$ has been shown to be suitable for large amplitude bubble oscillations. ${ }^{12}$ In this study, we combine the general form of the Keller-Miksis equation with the linear Voigt model for viscoelastic solids to study the dynamics of bubbles in soft tissue.

The importance of the inertial cavitation threshold has been addressed by many authors. In a medical context, severe bioeffects, including both thermal and nonthermal effects, may be induced or exacerbated by inertial cavitation during high intensity ultrasound insonations. When inertial cavitation occurs, strong nonlinear acoustic emissions can be detected. A sudden change in the emission signals from a bubble is often used to monitor the occurrence of inertial cavitation in experiments. ${ }^{13,14}$ In addition to the familiar second, third, and higher harmonics, bubbles may also generate subharmonic signals when they oscillate nonlinearly. Therefore, monitoring the generation of subharmonics can be used to detect inertial cavitation. Higher frequency emissions are very easily attenuated, and the signal that needs to be detected may become very weak. At the same time, the nonlinear propagation of ultrasound will also generate higher harmonics, and this can be a noise source for higher harmonic detection. Compared to harmonic detectors, subharmonic detectors have the advantages that the low frequency signal is less attenuated in soft tissue, and bubbles are the only sources generating subharmonics in soft tissue.

Subharmonic signals have been seen in experiments during cavitation events, but mechanisms for the generation of subharmonics are still not entirely clear. Possible explanations include that a single bubble will emit subharmonics when it breaks up, or interactions inside a bubble cloud can emit subharmonics, or chaotic oscillations of a single bubble will generate subharmonics. In this study, we focus on single bubble dynamics, and simply predict subharmonic signals from the chaotic oscillation of a single bubble. The effect of elasticity on inertial cavitation thresholds and subharmonic emissions will be investigated, providing data that may be useful for cavitation detection and bubble imaging.

\section{THEORY AND METHOD}

Consider a spherical bubble in an unbounded viscoelastic medium. The equation of continuity has the following form in a spherical coordinate system,

$$
\frac{\partial \rho}{\partial t}+\frac{\partial\left(\rho v_{r}\right)}{\partial r}+\frac{2 \rho v_{r}}{r}=0,
$$

where $\rho$ is the density, $v_{r}$ is the radial velocity, $t$ is time, and $r$ is the radial axis. Conservation of radial momentum for a spherically symmetric radial flow yields, ${ }^{15,16}$

$$
\rho\left(\frac{\partial v_{r}}{\partial t}+v_{r} \frac{\partial v_{r}}{\partial r}\right)=-\frac{\partial p}{\partial r}+\frac{\partial \tau_{r r}}{\partial r}+\frac{2}{r}\left[\tau_{r r}-\tau_{\theta \theta}\right],
$$

where $p$ is the pressure in the surrounding medium, and $\tau_{r r}$ and $\tau_{\theta \theta}$ are the stresses in the $r$ and $\theta$ directions, respectively. The boundary and initial conditions are:

$$
\begin{aligned}
& p=p_{g}-\frac{2 \sigma}{R}+\tau_{r r} \quad \text { at } r=R, \\
& p=p_{\infty} \quad \text { at } r=\infty, \\
& R=R_{0}, \quad \dot{R}=0 \quad \text { at } t=0,
\end{aligned}
$$

where $p_{g}$ is the gas pressure inside the bubble, $R$ is the position of the gas-tissue interface, the dot indicates the time derivative, $R_{0}$ is the bubble equilibrium radius, and $\sigma$ is the surface tension.

To derive the Keller-Miksis equation, which can account for the compressibility of the surrounding medium to first order, an asymptotic solution is employed in the near field and far field.

\section{A. Near field approximation}

In the near field $[r=O(R)]$, the effects of compression and expansion of the bubble are dominant, and the surrounding medium may be considered incompressible. From the Bernoulli integral (momentum equation), one can find the solution for the pressure distribution in the internal zone (near field),

$$
\begin{aligned}
v_{r}= & -\frac{\dot{R} R^{2}}{r^{2}} \\
p_{\text {in }}= & p_{a}-\rho_{0}\left(R \ddot{R}+\frac{3}{2} \dot{R}^{2}\right)+\frac{\rho_{0}}{r}\left(R^{2} \dot{R}\right)^{\prime}-\frac{\rho_{0}}{2} \frac{R^{4} \dot{R}^{2}}{r^{4}}+\left.\tau_{r r}\right|_{R} ^{r} \\
& +3 \int_{R}^{r} \frac{\tau_{r r}}{r} d r
\end{aligned}
$$

where $p_{a}$ is the pressure at the bubble surface, $\ddot{R}$ is the bubble wall acceleration, and, indicates the time derivative.

\section{B. Far field approximation}

In the far field $(r \gg R)$, the pressure fluctuations and the density fluctuations are small, and the stress components become negligible, as do the nonlinear convection terms. Ignoring these terms, the governing equation in the far field is essentially the linear acoustic equation. The solutions for the linear acoustic equation are

$$
\varphi_{\mathrm{ex}}=\frac{1}{r}\left[\psi_{1}\left(t-\frac{r}{c}\right)+\psi_{2}\left(t+\frac{r}{c}\right)\right],
$$




$$
p_{\mathrm{ex}}=p_{0}-\rho_{0} \frac{\partial \varphi_{\mathrm{ex}}}{\partial t}
$$

where $p_{0}$ is the static pressure, $\rho_{0}$ is density at equilibrium, $\varphi$ is the velocity potential, $\psi_{1}$ and $\psi_{2}$ characterize the outgoing and incident acoustic waves, respectively, and $c$ is the sound speed in the medium.

\section{Matching of the solutions}

To obtain the equation of the radial motion of bubbles for a given driving pressure and to take account of compressibility of the surrounding medium, we need to match the asymptotic solutions in the internal and external zones in the intermediate zone. For the internal solution, the intermediate zone is at $r \rightarrow \infty$, and for the external solution, the intermediate zone is at $r \rightarrow 0$. The matching conditions in the intermediate zone are the equality of the volumetric flow and of the pressure,

$$
\left.4 \pi r^{2} v_{r(\mathrm{in})}\right|_{r \rightarrow \infty}=\left.\left.4 \pi r^{2} v_{r(\mathrm{ex})}\right|_{r \rightarrow 0} \quad p_{\text {in }}\right|_{r \rightarrow \infty}=\left.p_{\mathrm{ex}}\right|_{r \rightarrow 0} .
$$

For the internal solution, shear stresses vanish as $r \rightarrow \infty$. By matching the solutions we finally get

$$
\begin{aligned}
R \ddot{R}+\frac{3}{2} R^{2}= & \frac{p_{a}-p_{0}}{\rho}+\frac{1}{c}\left[2 \psi_{2}^{\prime \prime}+f^{\prime \prime}\right]-\frac{\tau_{r r}(R, t)}{\rho} \\
& +\frac{3}{\rho} \int_{R}^{\infty} \frac{\tau_{r r}}{r} d r,
\end{aligned}
$$

where $f=R^{2} \dot{R}$, and $\psi_{2}$ is the incident wave. Note that the above equation includes $f^{\prime \prime}$, which will cause a third derivative of $R$. This was first noticed by Prosperetti et al. ${ }^{12}$ This third derivative can be eliminated by assuming that $f^{\prime \prime} / c$ is small, and evaluating the $f$ from the above-mentioned equation. Then the equation becomes

$$
R \ddot{R}+\frac{3}{2} R^{2}=\frac{p_{a}-p_{I}}{\rho},
$$

where $p_{I}$ is the pressure at infinity, $p_{I}=p_{0}-2 \rho / c \psi_{2}^{\prime \prime}$ $+\tau_{r r}(R, t)-3 \int_{R}^{\infty} \tau_{r r} / r d r$. This is the form of classic Rayleigh equation, indicating that the evaluation of $f$ from this equation is accurate to leading order. Equation (9) may be rewritten as

$$
\frac{f^{\prime}}{R}-\frac{1}{2} \frac{f^{2}}{R^{4}}=\frac{p_{a}-p_{I}}{\rho},
$$

then $f^{\prime}$ is evaluated as

$$
f^{\prime}=R\left[\frac{\dot{R}^{2}}{2}+\frac{p_{a}-p_{I}}{\rho}\right],
$$

Substitution of Eq. (11) into Eq. (8) results in the final equation,

$$
\begin{aligned}
(1 & \left.-\frac{\dot{R}}{c}\right) R \ddot{R}+\frac{3}{2}\left(1-\frac{\dot{R}}{3 c}\right) \dot{R}^{2} \\
& =\left(1+\frac{\dot{R}}{c}\right) \frac{p_{a}-p_{I}}{\rho}+\frac{R}{\rho c} \frac{d}{d t}\left[p_{a}-p_{I}\right],
\end{aligned}
$$

where $(2 \rho / c) \psi_{2}^{\prime \prime}=P_{A} g(t)$ is the driving pressure,

$$
p_{a}=p_{g}-\frac{2 \sigma}{R}+\tau_{r r}(R, t),
$$

and

$$
p_{a}-p_{I}=p_{g}-\frac{2 \sigma}{R}-p_{0}+\frac{2 \rho}{c} \psi_{2}^{\prime \prime}+3 \int_{R}^{\infty} \frac{\tau_{r r}}{r} d r
$$

\section{Evaluating stress components}

Since stress components will vanish in the far field, we then only evaluate $\tau_{r r}$ in the near field, i.e., in an incompressible material. Because soft tissue is viscoelastic, we need to choose a proper viscoelastic model to determine stresses. We choose the linear Voigt model for this study because it is a simple linear model and previous studies have shown that it is appropriate in the low megahertz frequency range., 9 More important, some experimental data for soft tissues are also available for this model. ${ }^{9,10}$ This also creates the potential for comparing the resulting predictions with the experimental measurements in vivo.

Because the material is incompressible, $\tau_{r r}=2\left(G \gamma_{r r}\right.$ $\left.+\mu \dot{\gamma}_{r r}\right)$, where $\gamma_{r r}$ is the strain, $\dot{\gamma}_{r r}$ is the strain rate with $\dot{\gamma}_{r r}=\partial u / \partial r, u$ is the velocity, and $G$ is the shear modulus (or rigidity). ${ }^{17}$ In the near field (near the bubble surface), $u=\left(R^{2} / r^{2}\right) \dot{R}$, therefore, $\gamma_{r r}=-\left(2 / 3 r^{3}\right)\left(R^{3}-R_{0}^{3}\right)$ and $\dot{\gamma}_{r r}=-\left(2 R^{2} / r^{3}\right) \dot{R}$.

Then, we have

$$
3 \int_{R}^{\infty} \frac{\tau_{r r}}{r} d r=-\left[\frac{4 G}{3 R^{3}}\left(R^{3}-R_{0}^{3}\right)+\frac{4 \mu \dot{R}}{R}\right],
$$

and

$$
\frac{d}{d t}\left(3 \int_{R}^{\infty} \frac{\tau_{r r}}{r} d r\right)=-4 G \frac{R_{0}^{3} \dot{R}}{R^{4}}-4 \mu\left(-\frac{\dot{R}^{2}}{R^{2}}+\frac{\ddot{R}}{R}\right) .
$$

Expanding $\left(p_{a}-p_{I}\right)$, finally we have

$$
\begin{aligned}
p_{a}-p_{I}= & p_{g}-\frac{2 \sigma}{R}-p_{0}+P_{A} g(t) \\
& -\left[\frac{4 G}{3 R^{3}}\left(R^{3}-R_{0}^{3}\right)+\frac{4 \mu \dot{R}}{R}\right],
\end{aligned}
$$

and 


$$
\begin{aligned}
\frac{d}{d t}\left(p_{a}-p_{I}\right) & \\
= & \frac{d}{d t}\left(p_{g}-\frac{2 \sigma}{R}-p_{0}+P_{A} g(t)\right)+\frac{d}{d t}\left[3 \int_{R}^{\infty} \frac{\tau_{r r}}{r} d r\right] \\
= & \frac{d p_{g}}{d t}+\frac{2 \sigma \dot{R}}{R^{2}}+P_{A} \frac{d g(t)}{d t}-4 G \frac{R_{0}^{3} \dot{R}}{R^{4}}-4 \mu\left(-\frac{\dot{R}^{2}}{R^{2}}+\frac{\ddot{R}}{R}\right) .
\end{aligned}
$$

Equations (12), (17), and (18a) provide the desired formulation describing the dynamics of gas bubbles in soft (i.e., viscoelastic) tissue. We note that this equation is actually just the Keller-Miksis equation with extra terms to account for the elasticity of soft tissue. The equation accounts for the compressibility of the surrounding medium to first order, and thus it is better suited than the Rayleigh-Plesset equation to simulate large amplitude bubble oscillations. The validity of this equation is limited to small Mach numbers. ${ }^{12}$

For the results presented here, the gas inside the bubble is assumed ideal, allowing the pressure to be estimated by use of a polytropic relation, $p_{g}=p_{g 0}\left(R_{0} / R\right)^{3 \kappa}$, where $\kappa$ is the polytropic index. In this case, Eq. (18a) may be written as:

$$
\begin{aligned}
\frac{d}{d t}\left(p_{a}-p_{I}\right)= & \left(\frac{2 \sigma}{R}-3 \kappa p_{g}\right) \frac{\dot{R}}{R}+P_{A} \frac{d g(t)}{d t}-4 G \frac{R_{0}^{3} \dot{R}}{R^{4}} \\
& -4 \mu\left(-\frac{\dot{R}^{2}}{R^{2}}+\frac{\ddot{R}}{R}\right) .
\end{aligned}
$$

\section{E. Analytical solutions}

Although Eq. (12) was specifically to investigate nonlinear bubble activity in tissue, it is instructive to consider the effects of the various physical parameters on bubble dynamics at low pressure amplitudes. An analytical solution to Eq. (12) may be obtained by assuming that the pulsation amplitude $R_{0} x(t)$, is small, making the usual substitutions of Eq. (19) into Eq. (12): ${ }^{18}$

$$
\begin{aligned}
& R=R_{0}(1+x), \quad U=R_{0} \dot{x}, \quad \dot{U}=R_{0} \ddot{x}, \\
& R^{-3 \kappa}=R_{0}^{-3 \kappa}(1-3 \kappa), \text { etc. },
\end{aligned}
$$

and recognizing that the term $i \omega R_{0} P_{A} e^{i \omega t} / c$ is to first order equivalent to the linear expression for the radiated pressure wave: ${ }^{15,18}$

$$
P_{\mathrm{sac}}=\frac{\rho \ddot{R} R_{0}}{\left(1-\frac{i \omega R_{0}}{c}\right)},
$$

The resulting equation has the form:

$$
m \ddot{x}+b \dot{x}+k x=-P_{A} e^{i \omega t},
$$

where the effective mass, $m$, total damping, $b_{\text {tot }}$, and stiffness, $k$, are given by

$$
m=\rho R_{0}^{2}+\frac{4 \mu R_{0}}{c},
$$

$$
\begin{aligned}
& b_{\text {tot }}=\left(3 \kappa P_{g 0}-\frac{2 \sigma}{R_{0}}+4 G\right) \frac{R_{0}}{c}+4 \mu \\
&+\frac{\omega R_{0} / c}{1+\left(\omega R_{0} / c\right)^{2}} \omega\left(\rho R_{0}^{2}\right), \\
& k=3 \kappa p_{g 0}-\frac{2 \sigma}{R_{0}}+4 G+\frac{\omega^{2}}{1+\left(\omega R_{0} / c\right)^{2}}\left(\rho R_{0}^{2}\right) .
\end{aligned}
$$

Notice that each term is greater than would be found from a purely linear analysis of a gas bubble in water. ${ }^{18}$ The effective mass contains a small additional increment due to the effect of viscosity. There are two additional damping terms, one arising from variation in the surface energy of the bubble (and directed opposite to the other damping terms), the other from the rigidity of the surrounding tissue, respectively. The rigidity of the tissue also contributes to the total stiffness of the system, as has been noted previously. ${ }^{1-6}$

Comparison of Eq. (21) with that of a damped harmonic oscillator:

$$
\ddot{x}+2 \beta_{\mathrm{tot}} \dot{x}+\omega_{0}^{2} x=\frac{-P_{A}}{m} e^{i \omega t},
$$

in which $\beta_{\text {tot }}$ is the total damping constant and $\omega_{0}$ is the natural frequency, allows identification of five components (viscous, thermal, acoustic, interfacial, and elastic) to the total damping, given by

$$
\begin{aligned}
\beta_{\mathrm{vis}}=b_{\mathrm{vis}} / 2 m & =2 \mu /\left(\rho R_{0}^{2}+\frac{4 \mu R_{0}}{c}\right), \\
\beta_{\mathrm{th}}=b_{\mathrm{th}} / 2 m & =3 \kappa p_{g 0} R_{0} /\left[2 c\left(\rho R_{0}^{2}+\frac{4 \mu R_{0}}{c}\right)\right] \\
& =3 \kappa p_{g 0} /\left(2 \rho c R_{0}+8 \mu\right), \\
\beta_{\mathrm{ac}}=b_{\mathrm{ac}} / 2 m & =\frac{\omega R_{0} / c}{1+\left(\omega R_{0} / c\right)^{2}} \frac{\omega}{2}\left(\rho R_{0}^{2}\right) /\left(\rho R_{0}^{2}+\right. \\
\beta_{\mathrm{int}}=b_{\mathrm{int}} / 2 m & =-\sigma /\left[c\left(\rho R_{0}^{2}+\frac{4 \mu R_{0}}{c}\right)\right] \\
& =-\sigma /\left(\rho c R_{0}^{2}+4 \mu R_{0}\right), \\
\beta_{\mathrm{el}}=b_{\mathrm{el}} / 2 m & =2 G R_{0} /\left[c\left(\rho R_{0}^{2}+\frac{4 \mu R_{0}}{c}\right)\right] \\
& =2 G /\left(\rho c R_{0}+4 \mu\right),
\end{aligned}
$$$$
\beta_{\mathrm{ac}}=b_{\mathrm{ac}} / 2 m=\frac{\omega R_{0} / c}{1+\left(\omega R_{0} / c\right)^{2}} \frac{\omega}{2}\left(\rho R_{0}^{2}\right) /\left(\rho R_{0}^{2}+\frac{4 \mu R_{0}}{c}\right),
$$

and the expression for the natural frequency:

$$
\begin{aligned}
\omega_{0}^{2}=k / m= & {\left[3 \kappa p_{g 0}-\frac{2 \sigma}{R_{0}}+4 G\right.} \\
& \left.+\frac{\omega^{2}}{1+\left(\omega R_{0} / c\right)^{2}}\left(\rho R_{0}^{2}\right)\right] /\left(\rho R_{0}^{2}+\frac{4 \mu R_{0}}{c}\right) .
\end{aligned}
$$

In this last expression, the contributions of the acoustic and viscous terms are important only for large bubbles, while the 
elastic term either dominates or is of the same order as the pressure term except for very small bubbles. Note that if the acoustic, viscous, and elastic terms are neglected, the wellknown expression for the resonance frequency of a gas bubble in liquid is recovered.

The scattering cross section may be defined as the ratio of the total acoustic power scattered by an object at a given frequency to the incoming acoustic intensity. ${ }^{17}$ For a spherical bubble oscillating at low amplitude, the expression for the scattering cross section is

$$
\sigma_{S}=\frac{4 \pi R_{0}^{2} \omega^{4}}{\left(\omega_{0}^{2}-\omega^{2}\right)^{2}+\beta_{\mathrm{tot}}^{2} \omega^{2}} .
$$

\section{F. Numerical solutions}

To obtain predictions for the nonlinear oscillation of a bubble in soft tissue, Eq. (12) must be solved numerically. Results for individual $R(t)$ curves, inertial cavitation thresholds, and subharmonic emissions are presented in the following. Determination of the cavitation thresholds requires selection of a threshold criterion. Several criteria for the inertial cavitation threshold have appeared in literature, e.g., $R_{\max } / R_{0}=2,{ }^{19,20} T_{\max }=5000 \mathrm{~K},{ }^{21}$ etc. In this study, we use $R_{\max } / R_{0}=2$ as the threshold criterion. This choice is consistent with the assumption that the air in the bubble expands and contracts adiabatically since the amplitude $R(t) / R_{0}$ is fairly insensitive to the thermodynamic processes within the bubble. ${ }^{22}$ For subharmonics, the relative strength of the emission with respect to that of the strongest frequency component emitted by a single bubble is shown. The reason to choose this relative strength is from consideration of experimental detection. After passing a preamp, whether a frequency component is detectable by a spectral analyzer or not depends on its strength relative to the strongest component. A frequency component is only detectable when this relative number is within the vertical resolution of the instrument. Otherwise, it will be suppressed as noise. The relative number is obtained by the following method: first the $R$ - $t$ curve is obtained, next the radiation pressure is determined by

$$
p_{\mathrm{sac}}(r, t)=\frac{\rho R}{r}\left(2 \dot{R}^{2}+R \ddot{R}\right),
$$

where $r$ is assumed to be a unit constant, and then frequency components are determined by FFT. The amplitude of the subharmonic is expressed in decibels relative to the maximum amplitude over all frequency components.

The following material properties are used in the current simulations: $\quad p_{0}=1.01 \times 10^{5} \mathrm{~Pa}, \quad \rho=1060 \mathrm{~kg} / \mathrm{m}^{3}, \quad c$ $=1540 \mathrm{~m} / \mathrm{s}$, and $\sigma=0.056 \mathrm{~N} / \mathrm{m}$ (the value for blood assumed by Apfel and Holland ${ }^{19}$ ). These parameters are chosen to be close to values appropriate to soft tissue. The actual properties of specific soft tissues may be slightly different from these values (except for surface tension, which is unknown), but the differences will generally be small. The polytropic index $\kappa=1.4$, a value appropriate for adiabatic oscillations of air bubbles. The rigidity and the viscosity of tissue are assumed to be $G=0,0.5,1.0$, and $1.5 \mathrm{MPa}$ and $\mu=0.015 \mathrm{~Pa}$ s. These values span the range obtained by pre-

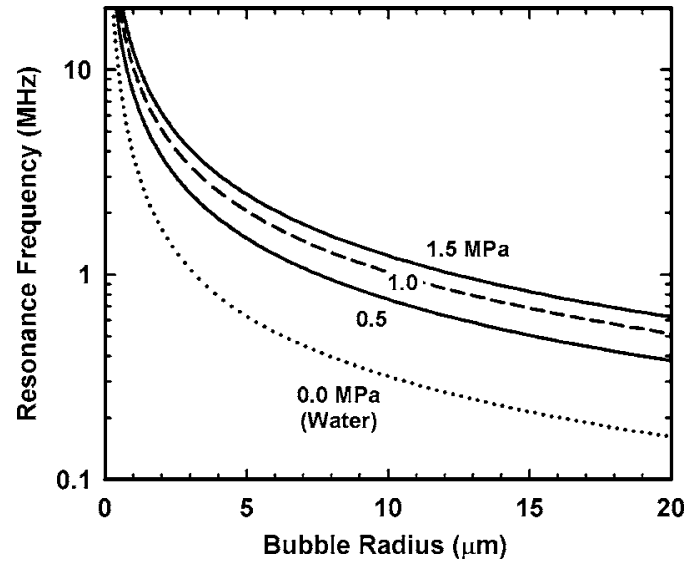

FIG. 1. Calculated values of linear resonance frequency for free air bubbles in water $(\cdots)$ and air bubbles surrounded by tissue having values of $G=1.5$ (upper-), $1.0(---)$, and $0.5 \mathrm{MPa}$ (lower-).

vious measurements, ${ }^{9,10}$ with the exception of $G=0$, which is included for comparison. The effect of viscosity has been investigated previously for a Newtonian medium. We will focus on the effects of elasticity on bubble motion in this study, but to allow easy comparison with previous work, results for water $(G=0, \mu=0.001)$ and blood $(G=0, \mu=0.005)$ are also presented. The driving frequencies studied are 1 and 3.5 MHz, which are frequently used in HIFU. All the bubble oscillations are solved for 30 cycles.

\section{RESULTS}

\section{A. Analytical results}

The following results were obtained for the case of airfilled bubbles in tissues having a modulus of rigidity equal to $0.5,1.0$, or $1.5 \mathrm{MPa}$ (as discussed earlier). In addition, results for either water or blood or both are presented for comparison. The effects of the surrounding tissue on resonance frequency, damping, and the scattering cross section for individual single bubbles will be illustrated in the following.

\section{Resonance frequency}

The undamped linear resonance frequency for bubbles larger than 1-2 $\mu \mathrm{m}$ and surrounded by viscoelastic tissue is dominated by the shear modulus $G$. For these bubbles, as shown in Fig. 1, $\omega_{0}$, increases approximately as the square root of $G$. The bottom curve in Fig. 1, labeled "Water," shows the resonance frequency for a free bubble with $G=0$. The three curves above it, for tissues with increasing values of $G$, demonstrate that the increase in stiffness provided by the tissue can increase the resonance frequency considerably. The effect of rigidity is much greater than the effect of surface tension, meaning that a larger bubble will exhibit a much greater stiffness than a free bubble of equivalent size. For example, the value of $\omega_{0}$, for a $5-\mu \mathrm{m}$ bubble is about $0.63 \mathrm{MHz}$, while replacing the water with tissue increases this value by a factor of $2.4,3.3$, and 4.0 times for the three rigidities studied here. Because these larger bubbles resonate at higher frequencies than free bubbles of equivalent size, they will tend to appear acoustically smaller than they actually are. 

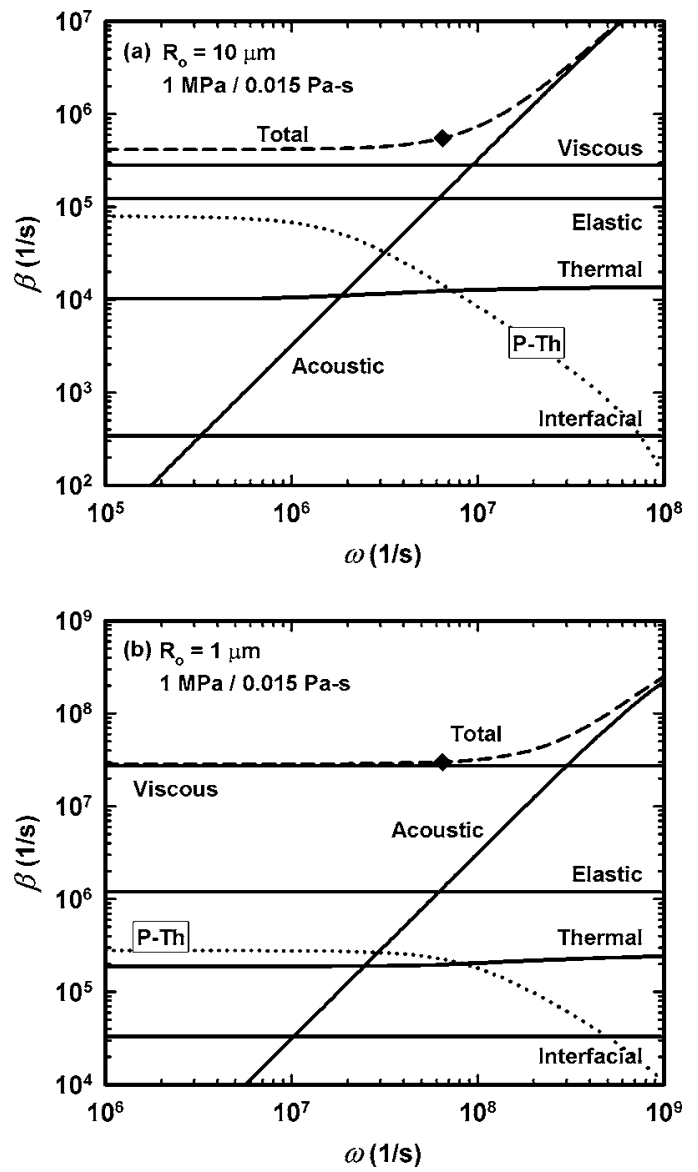

FIG. 2. Dimensional linear damping constants vs radial frequency for equilibrium bubble radii of (a) $10 \mu \mathrm{m}$ and (b) $1 \mu \mathrm{m}$, surrounded by tissue with $G=1.0 \mathrm{MPa}$ and $\mu=0.015 \mathrm{Pas}$; resonance radius $(\downarrow)$, thermal damping constant given by Prosperetti-Ref. $25(\cdots)$.

\section{Linear damping coefficients}

Five sources of damping for bubbles surrounding by viscoelastic tissue were identified earlier and quantified by Eq. (23). Two of these expressions, for viscous and acoustic damping, are identical to those given by Prosperetti ${ }^{18}$ for a gas bubble in liquid, see Eqs. (23a) and (23c), respectively. The expression for thermal damping, Eq. (23b), differs markedly from those given by either Prosperetti ${ }^{18}$ or Eller, ${ }^{23}$ which is not surprising given the assumption of a polytropic pressure-volume relation for the gas. However, the numerical values obtained for bubbles smaller than the linear resonance radius are remarkably similar, often to within a few percent, of those obtained using more exact theories. ${ }^{18,23}$ The two additional damping terms, due to the surface energy of the bubble and the rigidity of the surrounding tissue, see Eqs. (23d) and (23e), respectively, have a form similar to that for thermal damping, Eq. (23b). It is worth noting that a combination of four of the damping terms, Eqs. (23b)-(23e), is proportional to the stiffness, and thus also to the resonance frequency, of the system.

The results of calculations for the damping constants as a function of radial frequency are shown in Fig. 2 for bubble radii of 1 and $10 \mu \mathrm{m}$, assuming $G=1.0 \mathrm{MPa}$ and $\mu$ $=0.015 \mathrm{~Pa}$ s. Because the term for interfacial tension is negative, only its magnitude has been plotted here. In any case,
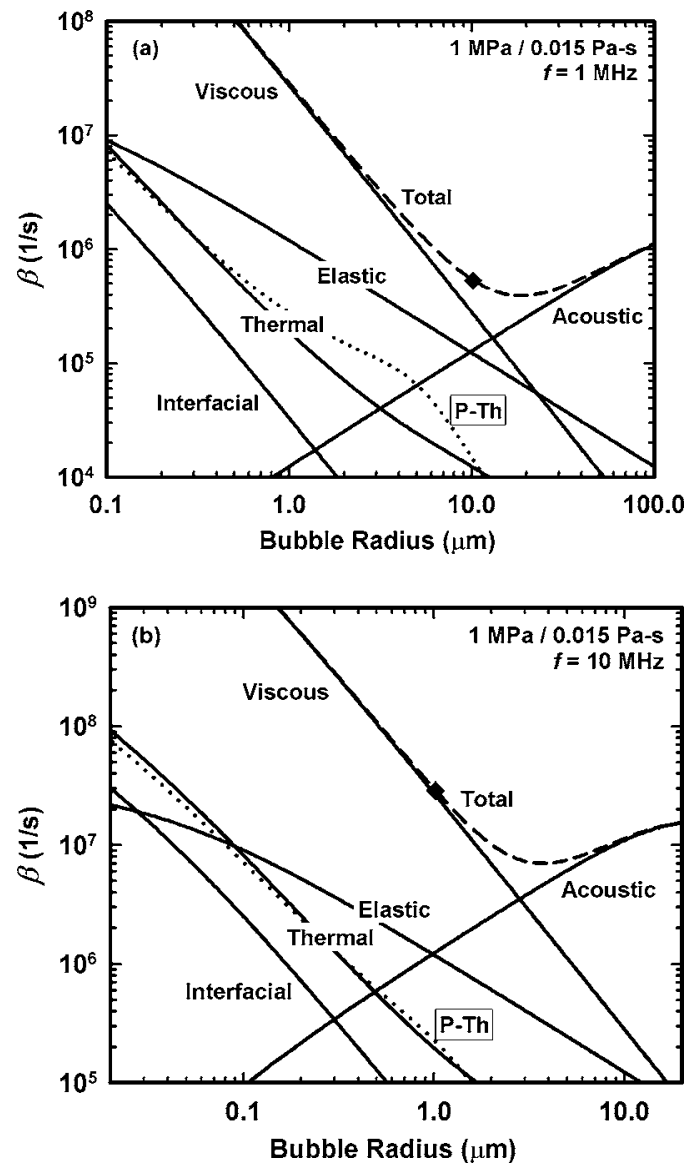

FIG. 3. Dimensional linear damping constants vs equilibrium bubble radius for radial frequencies of (a) $1 \mathrm{MHz}$ and (b) $10 \mathrm{MHz}$, for air bubbles surrounded by tissue with $G=1.0 \mathrm{MPa}$ and $\mu=0.015 \mathrm{Pas}$; resonance radius $(\diamond)$, thermal damping constant given by Prosperetti-Ref. $25(\cdots)$.

the contribution of $\beta_{\text {int }}$ to $\beta_{\text {tot }}$ is quite modest. The figures have been drawn in such a way as to allow easy comparison with previous results for free ${ }^{18,23}$ and encapsulated bubbles. ${ }^{17}$ Due to the high value of $\mu$, the total damping is dominated by $\beta_{\mathrm{vis}}$ for frequencies less than $\omega_{0}$ and $R_{0}<10 \mu \mathrm{m}$, while the acoustic term $\beta_{\mathrm{ac}}$ dominates at higher frequencies. The elastic term becomes increasingly important as $R_{0}$ increases, with $\beta_{\mathrm{el}} \sim \beta_{\mathrm{vis}}$ at $R_{0}=30 \mu \mathrm{m}$. As noted earlier, the expression for $\beta_{\text {th }}$ differs from those obtained using more exact approaches. This is reflected in both the shape and the magnitude of the curves labeled "Thermal" in Fig. 2, as may be seen by comparison with the dotted curves labeled "P-Th," obtained using the theory of Prosperetti. ${ }^{18}$ Thus, while $\beta_{\mathrm{th}}$ $<\beta_{\mathrm{el}}$ for all values of $R_{0}$ when employing the polytropic assumption (as is done here), $\beta_{\text {th }}$ would come to dominate other sources of damping for $R_{0}>30 \mu \mathrm{m}$ and $\omega<\omega_{0}$ in a more rigorous treatment of thermal effects.

The results of calculations for damping constants as a function of radius are given in Fig. 3 for frequencies of 1 and $10 \mathrm{MHz}$, again assuming $G=1.0 \mathrm{MPa}$ and $\mu=0.015 \mathrm{~Pa}$. The total damping is dominated by $\beta_{\text {vis }}$ for bubbles smaller than the linear resonance radius, while $\beta_{\mathrm{ac}}$ dominates at larger sizes. The contribution of $\beta_{\mathrm{el}}$ is never more than about $20 \%$ of $\beta_{\text {tot }}$, which occurs near the resonance radius at $1 \mathrm{MHz}$, see Fig. 3(a). For frequencies above about $1 \mathrm{MHz}$ and radii less than the resonance size, the values for $\beta_{\text {th }}$ 

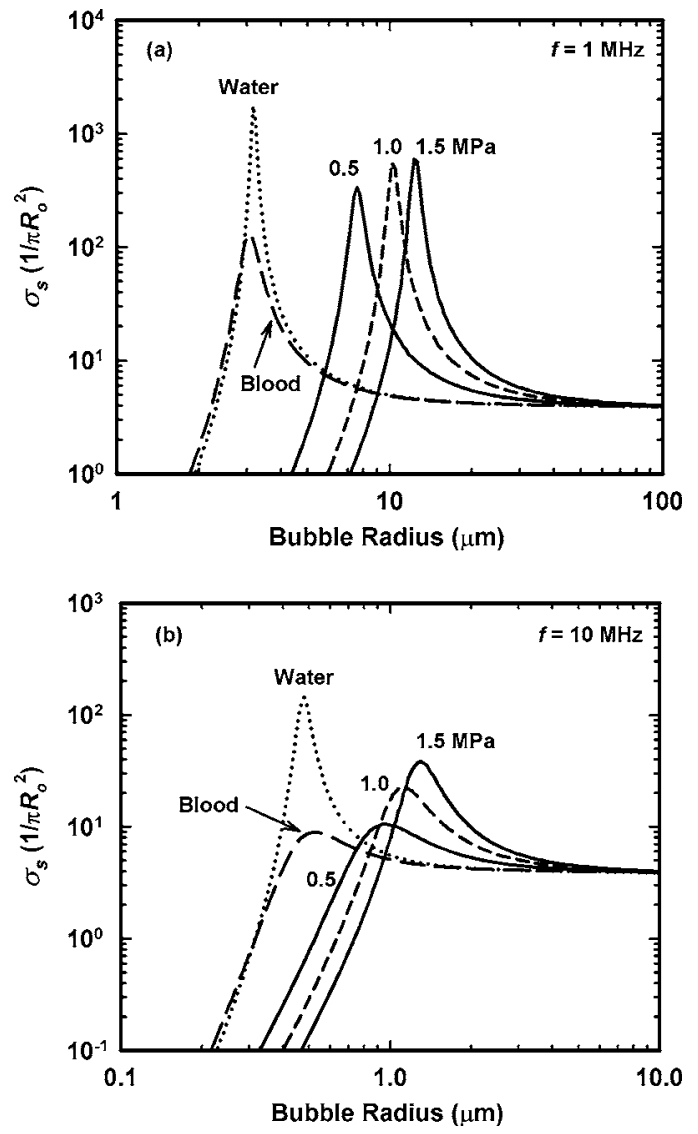

FIG. 4. Linear scattering cross sections vs equilibrium bubble radius for frequencies of (a) $1 \mathrm{MHz}$ and (b) $10 \mathrm{MHz}$, for air bubbles surrounded by tissue having values of $G=1.5$ (right-), $1.0(---)$, and $0.5 \mathrm{MPa}$ (left-) with $\mu=0.015 \mathrm{~Pa} \mathrm{~s}$, and for water $(\cdots)$ and blood $(---)$.

calculated using Eq. (23b) and the theory of Prosperetti, ${ }^{18}$ (dotted curves labeled "P-Th") agree rather closely, although the contribution of $\beta_{\text {th }}$ to $\beta_{\text {tot }}$ is not significant. The contribution of $\beta_{\text {int }}$ to $\beta_{\text {tot }}$ is also trivial.

\section{Scattering cross section}

Calculated values of the linear scattering cross sections of individual air bubbles, normalized to their respective geometrical cross sections, are given in Fig. 4 for driving frequencies of 1 and $10 \mathrm{MHz}$. The strong effect of tissue elasticity (i.e., $G$ ) is apparent in these results, causing the resonance peaks to shift to bubble radii two to four times larger than for the resonance peak in water. Even though they are larger, the cross sections for bubbles surrounded by tissue are less, and sometimes much less, than for resonant bubbles in water. It is also seen that the curves broaden and diminish as either the rigidity $G$ decreases or the frequency $f$ increases, indicating that bubbles in tissue may be more difficult to detect acoustically than are bubbles in water at the same frequency. The cross sections for blood exhibit maxima at about the same radii as for water, but their magnitudes are less due to the higher viscosity of that fluid.

\section{B. Numerical results}

In this part of the paper, simulation results will be given for numerical solutions of Eq. (12). The effects of elasticity
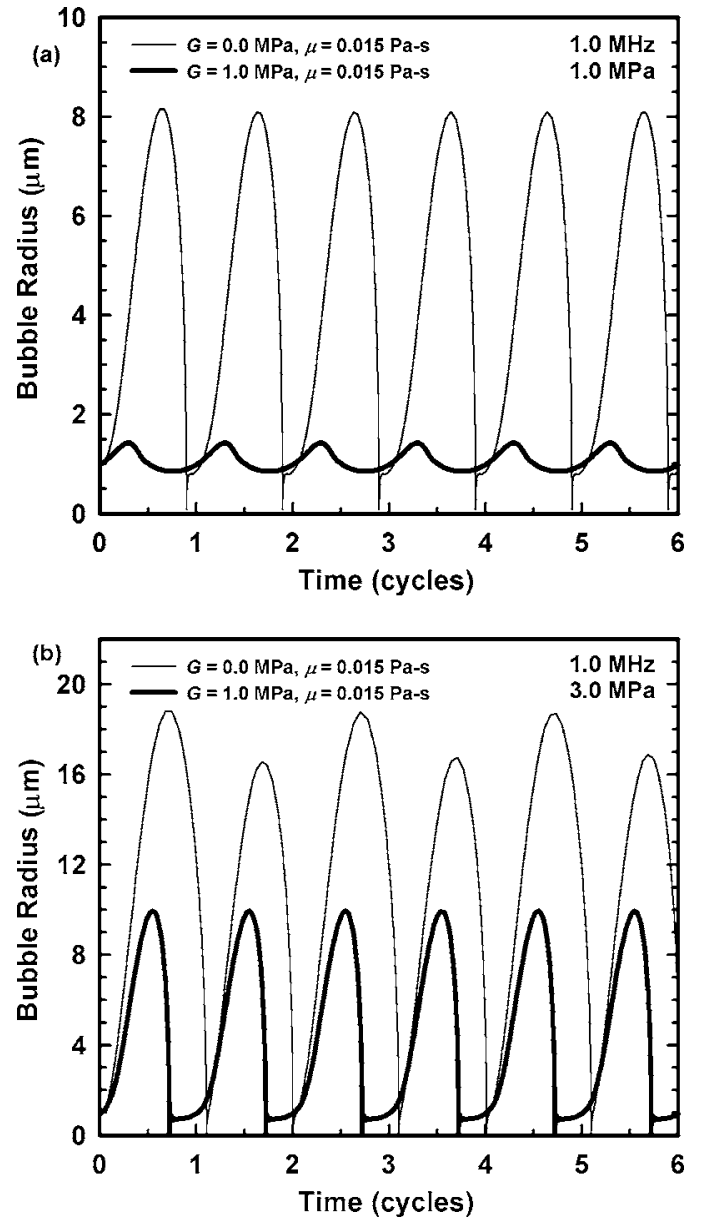

FIG. 5. A comparison of radial responses for $1-\mu \mathrm{m}$ bubbles driven by a $1-\mathrm{MHz}$ pulse at (a) $1 \mathrm{MPa}$ and (b) $3 \mathrm{MPa}$, for $G=0$ (thin line), and $G=1.0 \mathrm{MPa}$ (thick line); the viscosity was fixed at $0.015 \mathrm{~Pa}$.

on $R$ - $t$ curves will be examined first. Next, initial cavitation thresholds will be presented for different elasticities and compared to the result in water. Finally, maps of the strength of subharmonic signals emitted by oscillating bubbles will highlight a common way to detect cavitation and will illustrate the ranges of bubble radii that may be easily detected using this method.

\section{The effect of elasticity on $R(t)$ curves}

Examples of $R(t)$ curves for a $1-\mu \mathrm{m}$ bubble oscillating under $1-\mathrm{MHz}$ driving pressures of 1 and $3 \mathrm{MPa}$ are shown in Figs. 5(a) and 5(b), respectively. The viscosity is fixed at $0.015 \mathrm{Pas}$ and the rigidity is chosen as 0 and 1.0 MPa. The effect of the elasticity is very obvious that it greatly reduces the amplitude, and hence the nonlinearity, of the oscillation. At $1 \mathrm{MPa}$, the amplitude of the oscillation is much smaller when elasticity is included. When the driving pressure increases to $3 \mathrm{MPa}$, the amplitude of the oscillation with nonzero elasticity is still smaller than that with zero elasticity, but the difference between the two cases is less. This indicates that the effect of elasticity will be less when the driving pressure is strong. Another feature which is worthy of comment is that for the zero-elasticity case, the bubble oscillation approaches a steady-state resonance ${ }^{22,24}$ of order $2 / 2$, an example of period doubling and an indication of the start of 

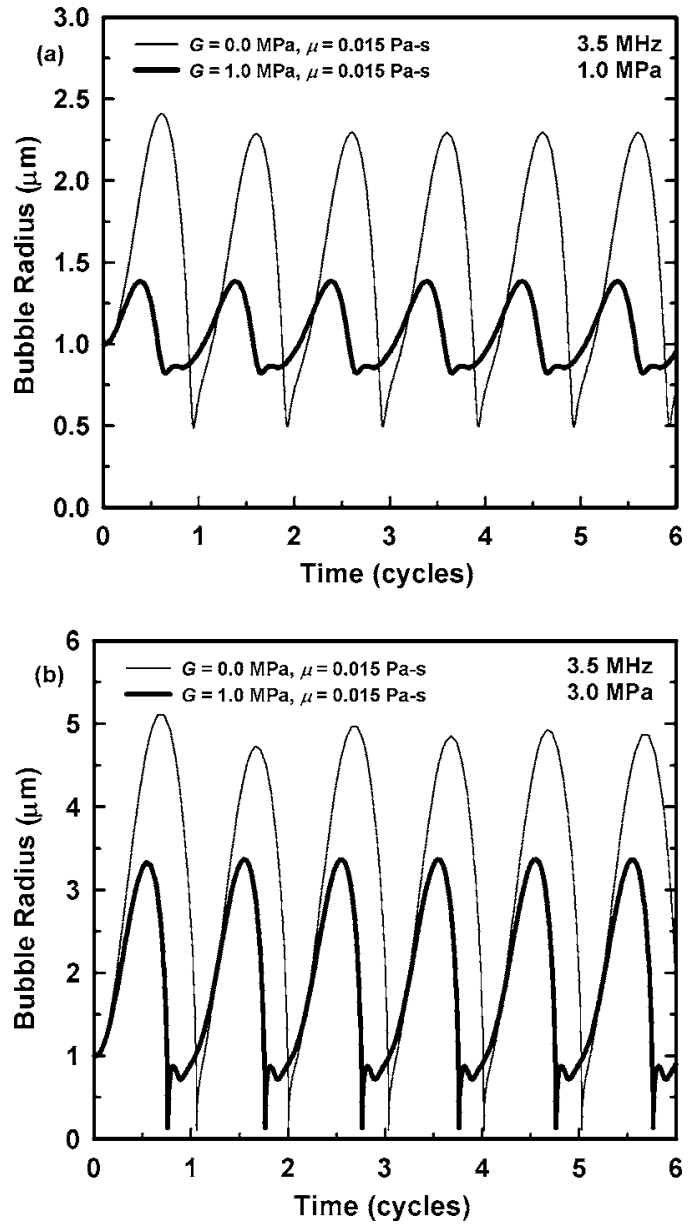

FIG. 6. A comparison of radial responses for $1-\mu \mathrm{m}$ bubbles driven by a 3.5-MHz pulse at (a) $1 \mathrm{MPa}$ and (b) $3 \mathrm{MPa}$, for $G=0$ (thin line), and $G$ $=1.0 \mathrm{MPa}$ (thick line); the viscosity was fixed at $0.015 \mathrm{Pas}$.

chaotic oscillation and very strong nonlinearity. For the nonzero-elasticity case, no period doubling is observed under this driving pressure, although the inertial collapses are still very strong. Clearly, the presence of the elasticity has reduced or eliminated some nonlinear components of the bubble oscillation.

Figure 6 shows examples of $R(t)$ curves for a $1-\mu \mathrm{m}$ bubble driven by (a) $1 \mathrm{MPa}$ and (b) $3 \mathrm{MPa}$ at $3.5 \mathrm{MHz}$. Similar conclusions as those at $1 \mathrm{MHz}$ can be drawn from these results. For the same driving pressure and the same size bubble, the nonlinearity appears to be weaker at the higher frequency because no period doubling is observed. Figure 7 shows the results for a $5-\mu \mathrm{m}$ bubble at $1 \mathrm{MHz}$ driven by (a) $1 \mathrm{MPa}$ and (b) $3 \mathrm{MPa}$. For zero elasticity, bubbles oscillate with larger amplitudes during the first few cycles than that for the nonzero-elasticity case. Interestingly, after the initial state, the presence of the elasticity increases the amplitude of oscillation in both cases. In Fig. 7(a), at zero-elasticity, the bubble oscillation exhibits strong nonlinear behavior by a decrease at the average radius, and the presence of elasticity recovers the linear oscillation around the bubble equilibrium radius. In Fig. 7(b), although the oscillation amplitude is smaller at zero-elasticity case, the oscillation itself becomes chaotic, while it is periodic when elasticity is included. Compared to bubbles of smaller size, a stronger nonlinearity is
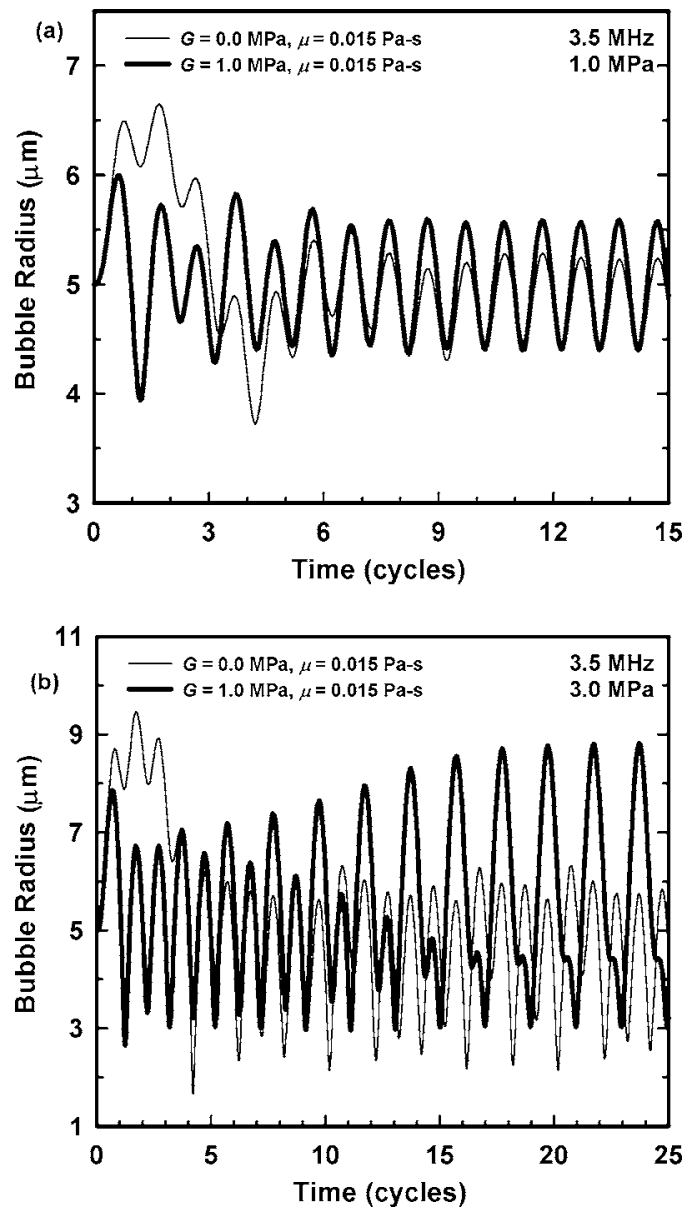

FIG. 7. A comparison of radial responses for $5-\mu \mathrm{m}$ bubbles driven by a 3.5-MHz pulse at (a) $1 \mathrm{MPa}$ and (b) $3 \mathrm{MPa}$, for $G=0$ (thin line), and $G$ $=1.0 \mathrm{MPa}$ (thick line); the viscosity was fixed at $0.015 \mathrm{~Pa}$.

observed, which is indicated by the approach to chaotic oscillations. Certainly, the nonlinearity does not always increase when the bubble size increases.

\section{The effect of elasticity on the inertial cavitation threshold}

In Fig. 8, predicted inertial cavitation thresholds are shown for a driving frequency of $1 \mathrm{MHz}, G=0,0.5,1.0$, and $1.5 \mathrm{MPa}$, and $\mu=0.015 \mathrm{Pas}$. The thresholds in water and

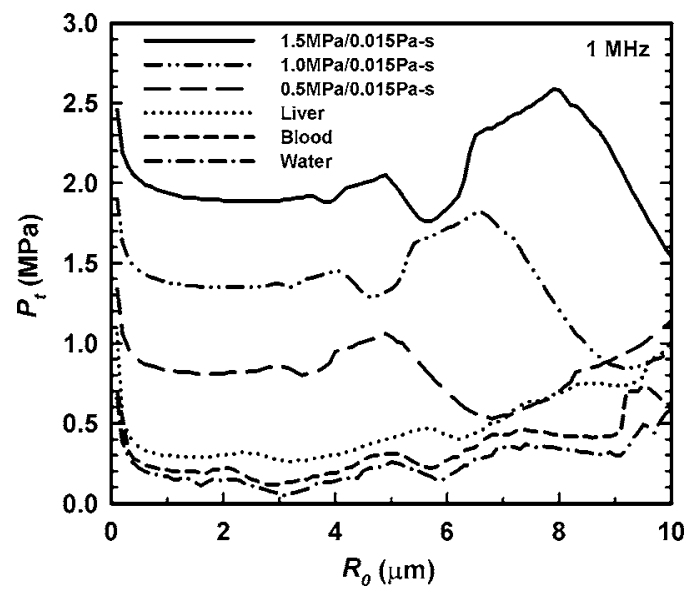

FIG. 8. Predicted thresholds for inertial cavitation at $1 \mathrm{MHz}$. 


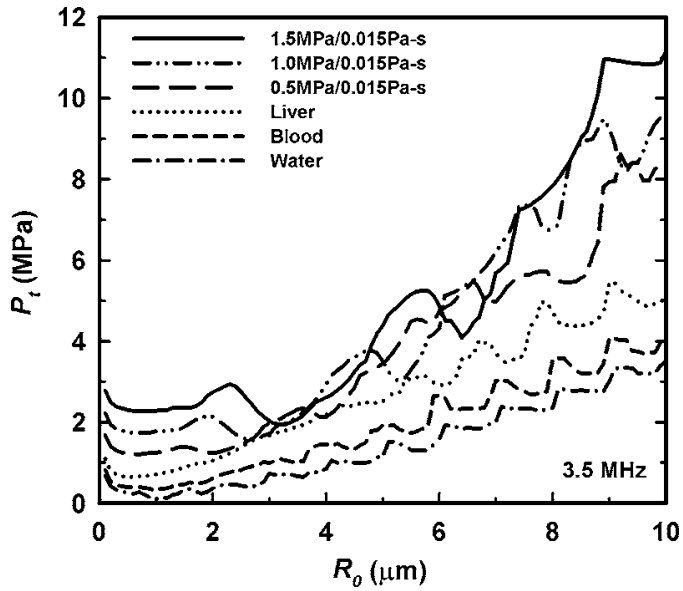

FIG. 9. Predicted thresholds for inertial cavitation at 3.5 MHz.

blood are also shown in the plot for reference. The effect of elasticity on the threshold is obvious. Threshold values increase as the elasticity increases, as expected based on the results for the $R(t)$ curves given earlier. For nonzero elasticity, the thresholds have significant structure that deserves comment. For example, at $G=1.5 \mathrm{MPa}$, for small bubble sizes, the threshold value is consistent with the Blake threshold. As the bubble size increases, the threshold value reaches a minimum value and then slowly rises. At around $4 \mu \mathrm{m}$, the threshold value suddenly drops and then comes back. The same shape occurs at around $6 \mu \mathrm{m}$, and another drop in threshold occurs around $8 \mu \mathrm{m}$. The lowest threshold attained following each drop is less than the preceding minimum at a smaller bubble size. Similar structures are observed for the lower elasticity cases, the only difference being that these drops occur at different positions. This resonant structure appears to be related to the fractional-order subharmonic resonance minima described previously. ${ }^{22}$ The positions of the minima are determined by the elasticity of the surrounding medium, shifting to larger radii as $G$ increases.

Figure 9 shows the same thresholds but at a driving frequency of 3.5 MHz. At this higher frequency, the threshold values are greater than at $1 \mathrm{MHz}$, and the increase in thresholds for larger bubble sizes is much faster than that at $1 \mathrm{MHz}$. The resonant structures appearing in the nonzeroelasticity cases at $1 \mathrm{MHz}$ are also observed on these threshold curves. The resonance structure differs significantly however in that the minimum values attained following the drops in the curves at $3.5 \mathrm{MHz}$ are not less than the immediately preceding minima.

The effect of viscosity on the threshold can be observed by comparing the threshold for $G=0 \mathrm{MPa}$ and those of blood and water (the only difference among the three is the value of viscosity, $\mu=0.015,0.005$, and $0.001 \mathrm{~Pa} \mathrm{~s}$, respectively). In comparing, we conclude that thresholds increase and have less structure as viscosity increases.

\section{The map of the strength of subharmonics}

Figure 10 shows subharmonic emissions at a driving frequency of $1 \mathrm{MHz}$ as a function of driving pressure and bubble equilibrium radius. Results for tissues with $G=0,0.5$, and 1.0 $\mathrm{MPa}$ and $\mu=0.015 \mathrm{Pas}$ are shown, with the result for blood also shown for reference $(G=0 \mathrm{MPa}, \mu$ $=0.005 \mathrm{Pas}$ ). We will define a subharmonic signal as "strong" when it is greater than $-30 \mathrm{~dB}$ because above this level, the subharmonic signal can be easily detected by an instrument with an 8-bit dynamic range. From Figs. $10(\mathrm{a})-10(\mathrm{c})$, it is seen that as the elasticity increases, the strong subharmonic signal region moves toward larger bubble sizes and higher driving pressures. The strong subharmonic signal region generally lies above the corresponding inertial cavitation threshold. For small values of elasticity, the inertial threshold coincides with the lower boundary of the strong subharmonic emission region better than does that for higher elasticity cases. This result is expected because the subharmonic signal investigated here arises from chaotic bubble oscillations, and chaotic oscillations usually occur after the bubble motion becomes strongly nonlinear. In comparing the map for blood and that for tissue with $G$ $=0 \mathrm{MPa}$, we find that the strong subharmonic signal region becomes smaller when the viscosity increases. The contour lines on these plots indicate the boundaries between regions in which the maximum emission occurs at a particular frequency. The results show that all strong subharmonic emissions occur only when the fundamental frequency component has the maximum emission level.

Figure 11 shows results for the same cases as in Fig. 10, but at a driving frequency of $3.5 \mathrm{MHz}$. As the elasticity increases, the strong subharmonic emission region shrinks and moves toward higher pressure amplitudes. Significantly perhaps, the relative signal also seems stronger. Compared to results at $1 \mathrm{MHz}$, the strong subharmonic emission region at 3.5 MHz is much smaller and is limited to the small bubble region. This limitation to the small bubble region probably is related to the resonance structure of these bubble responses. At $3.5 \mathrm{MHz}$, the linear bubble resonance size is smaller than that at $1 \mathrm{MHz}(3.07$ vs $10.67 \mu \mathrm{m})$. Again, all strongemission regions are above the inertial cavitation thresholds for the corresponding elasticity, and strong subharmonic emissions occur only when the fundamental frequency component has the maximum emission level.

\section{DISCUSSION AND CONCLUSIONS}

In this study, we developed a theoretical model for the pulsations of gas bubbles in simple linear viscoelastic solids and presented some potentially useful results for the case of soft tissues. As pointed out in the text, although the model is simple, it is consistent with experimental data taken for some soft tissues. However, at high intensity, bubble oscillations are strongly nonlinear. Although strong nonlinear oscillations do not automatically imply that a nonlinear viscoelastic model is necessary to describe the bubble motion, the suitability of this linear model also remains unclear. As a matter of fact, there is little evidence that the strain-stress relation in tissue is not linear. Even though the change in bubble radius is significant compared to its initial dimension, the overall strain in the tissue could still be considered small if this change were compared to the dimension of the soft tissue. Use of a different viscoelastic model certainly would result in different predictions, but the sparse measurement data at 

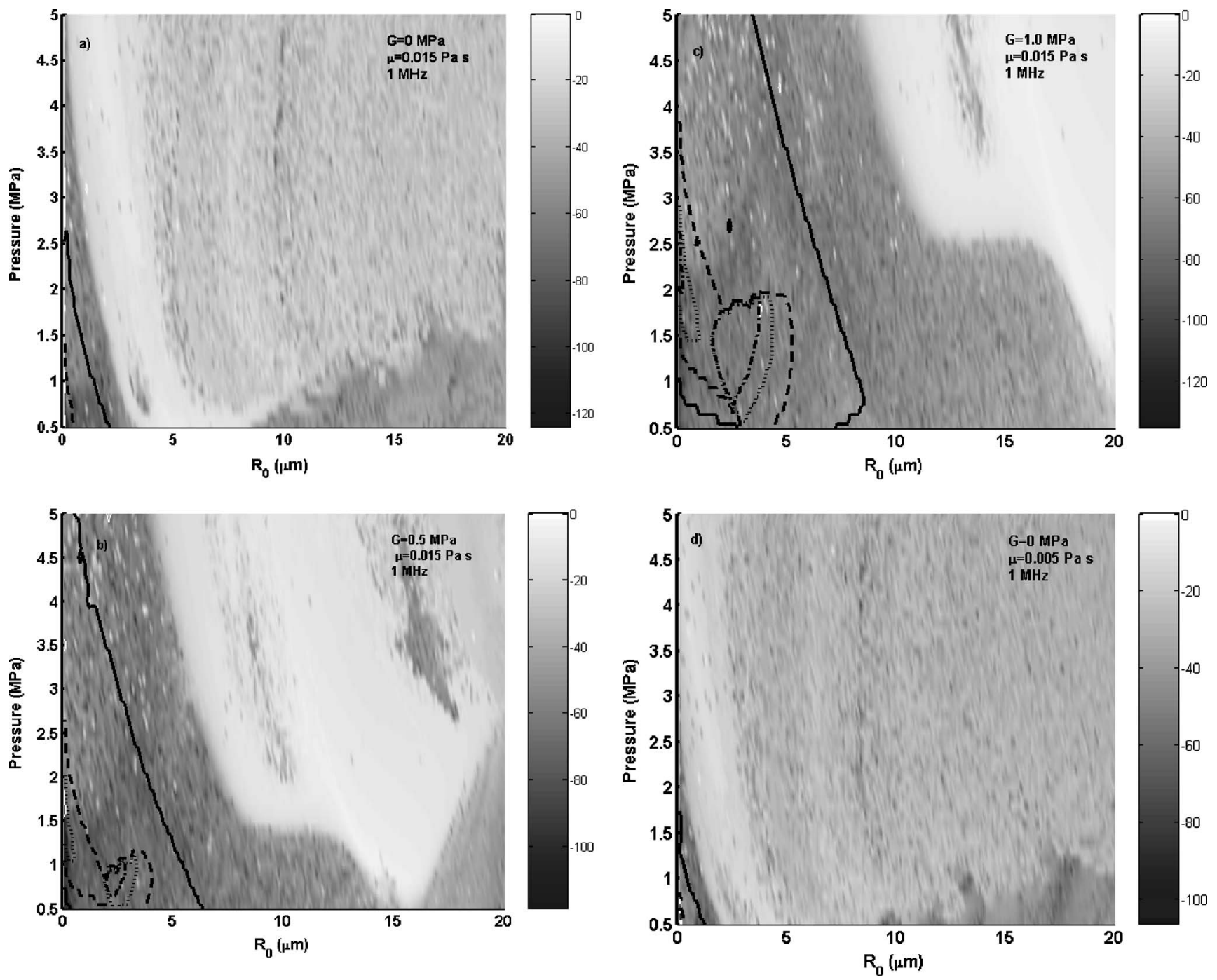

FIG. 10. Subharmonic emissions in $\mathrm{dB}$ relative to the peak emission at a driving frequency of $1 \mathrm{MHz}$ as a function of driving pressure and bubble equilibrium radius for three "tissues:" (a) $G=0 \mathrm{MPa}$ and $\mu=0.015 \mathrm{~Pa} \mathrm{~s}$; (b) $G=0.5, \mu=0.015$; (c) $G=1.0, \mu=0.015$; and for (d) blood, $G=0, \mu=0.005$. The contour lines indicate the boundaries between regions in which maximum emissions occur only at one frequency component: fundamental (right-most region) and second harmonic (solid line); second harmonic and third harmonic (dashed line); third harmonic and fourth harmonic (dotted line); fourth harmonic and fifth harmonic (dash-dot line).

megahertz frequencies limits the study of verified viscoelastic models for soft tissue. Also, the present study provides predictions that can be examined experimentally. Verification of this model will be pursued in the future.

Linear analysis of this inherently nonlinear formulation provided analytical predictions of bubble responses to insonation at low pressure amplitudes. The result for resonance frequency increases as the modulus of rigidity increases, as was expected based on previous work. ${ }^{17}$ The results for damping constants showed that the viscosity of the tissue tends to dominate either thermal or elastic damping for bubbles smaller than $\sim 30 \mu \mathrm{m}$ for frequencies smaller than the linear resonance size, while acoustic damping predominates at higher frequencies. It is also expected that thermal damping would dominate other sources of damping for $R_{0}$ $>30 \mu \mathrm{m}$ and $\omega<\omega_{0}$ in a more rigorous treatment of thermal effects than is given by the polytropic assumption used here. The peaks in the curves for scattering cross section shift to larger radii as the rigidity increases due to the increase in resonance frequency, although their magnitudes are less, and sometimes much less, than is the case for resonant bubbles in water. This is consistent with the numerical results used in producing Figs. 10 and 11.

The effect of elasticity on bubble dynamics was investigated in some detail. Overall, the presence of the elasticity in a bubble dynamics equation will reduce, sometimes greatly, the nonlinearity of bubble oscillations. As might be expected, the inertial cavitation threshold was shown to be greater in tissue than in liquids such as water or blood, in contrast to the assumptions underlying the mechanical index. ${ }^{19,25}$ This result should prove useful for understanding the prevalence of potentially damaging inertial cavitation in vivo. This will be the subject of a later study.

Subharmonic emissions from an oscillating viscoelastic bubble were also studied. Since soft tissue generally is not transparent, detecting acoustic emissions is often the best way to gain information about a bubble. A passive or active cavitation detector system can easily provide information about bubbles inside soft tissues. When interpreting such re- 

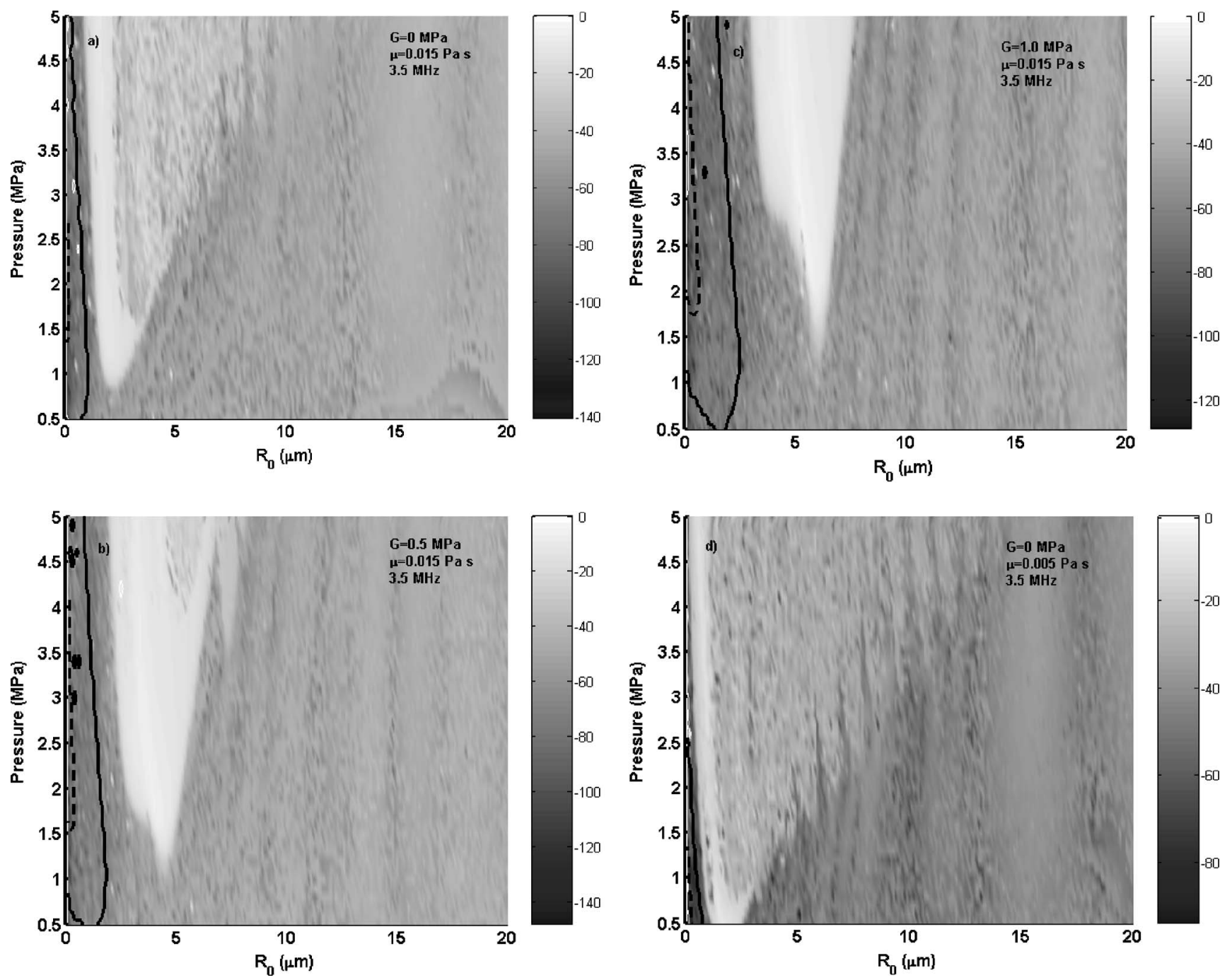

FIG. 11. Subharmonic emissions in $\mathrm{dB}$ relative to the peak emission at a driving frequency of $3.5 \mathrm{MHz}$ as a function of driving pressure and bubble equilibrium radius for three "tissues:" (a) $G=0 \mathrm{MPa}$ and $\mu=0.015 \mathrm{~Pa} \mathrm{~s}$; (b) $G=0.5, \mu=0.015$; (c) $G=1.0, \mu=0.015$; and for (d) blood, $G=0, \mu=0.005$. The contour lines indicate the boundaries between regions in which maximum emissions occur only at one frequency component: fundamental (right-most region) and second harmonic (solid line); second harmonic and third harmonic (dashed line); third harmonic and fourth harmonic (dotted line); fourth harmonic and fifth harmonic (dash-dot line).

sults however, it will be important to keep in mind that the sizes of the bubbles detected will be greater than estimated using linear theory for water.

Shape oscillations and rectified diffusion are not considered in this study, although they are very important to predicting the stability and equilibrium size of a bubble. ${ }^{26}$ Shape oscillations could also generate larger stresses that could cause severe mechanical damage to soft tissue. Rectified diffusion will change the equilibrium size of a bubble, and the bubble motion can thereby be greatly affected. These effects will be the focus of future studies.

\section{ACKNOWLEDGMENTS}

The suggestions and support of the Biomedical Acoustic group at NCPA are gratefully acknowledged. This work was supported by Award No. DAMD17-02-2-0014, administered by the US Army Medical Research Acquisition activity, Fort Detrick, MD. The information contained herein does not nec- essarily reflect the position or policy of the US government, and no official endorsement should be inferred.

${ }^{1}$ H. S. Fogler and J. D. Goddard, "Collapse of spherical cavities in viscoelastic fluids," Phys. Fluids 13, 1135-1141 (1970).

${ }^{2}$ I. Tanasawa and W. J. Yang, "Dynamic behavior of a gas bubble in viscoelastic liquids," J. Appl. Phys. 41, 4526-4531 (1970).

${ }^{3}$ A. Shima, T. Tsujino, and H. Nanjo, "Nonlinear oscillations of gas bubbles in viscoelastic fluids," Ultrasonics 24, 142-147 (1986).

${ }^{4}$ C. Kim, "Collapse of spherical bubbles in Maxwell fluids," J. NonNewtonian Fluid Mech. 55, 33-58 (1994).

${ }^{5}$ V. N. Alekseev and S. A. Rybak, "The behavior of gas bubbles in insonated biological tissues," Acoust. Phys. 44, 243-247 (1998).

${ }^{6} \mathrm{~J}$. S. Allen and R. A. Roy, "Dynamics of gas bubbles in viscoelastic fluids. I. Linear viscoelasticity," J. Acoust. Soc. Am. 107, 3167-3178 (2000).

${ }^{7}$ J. S. Allen and R. A. Roy, "Dynamics of gas bubbles in viscoelastic fluids. II. Nonlinear viscoelasticity," J. Acoust. Soc. Am. 108, 1640-1650 (2000).

${ }^{8}$ C. C. Church, "Spontaneous homogeneous nucleation, inertial cavitation and the safety of diagnostic ultrasound," Ultrasound Med. Biol. 28, 13491364 (2002).

${ }^{9}$ L. A. Frizzell, E. L. Carstensen, and J. F. Dyro, "Shear properties of mammalian tissues at low megahertz frequencies," J. Acoust. Soc. Am. 60, 1409-1411 (1977). 
${ }^{10}$ E. L. Madsen, H. J. Sathoff, and H. J. Zagzebski, "Ultrasonic shear wave properties of soft tissues and tissuelike materials," J. Acoust. Soc. Am. 74, 1346-1355 (1983).

${ }^{11}$ J. B. Keller and M. J. Miksis, "Bubble oscillations of large amplitude," J. Acoust. Soc. Am. 68, 628-633 (1980).

${ }^{12}$ A. Prosperetti and A. Lezzi, "Bubble dynamics in a compressible liquid. 1. First order theory," J. Fluid Mech. 168, 457-478 (1986).

${ }^{13}$ R. A. Roy, S. I. Madanshetty, and R. E. Apfel, "An acoustic backscattering technique for the detection of transient cavitation produced by microsecond pulses of ultrasound," J. Acoust. Soc. Am. 87, 2451-2458 (1990).

${ }^{14}$ ANSI Technical Report, "Bubble Detection and Cavitation Monitoring," American National Standards Institute, Document ANSI S1.24 TR-2002, 2002.

${ }^{15}$ L. D. Landau and E. M. Lifshitz, Fluid Mechanics (Pergamon, Oxford, 1959), Chap. II.

${ }^{16}$ A. Prosperetti, "A generalization of the Rayleigh-Plesset equation of bubble dynamics," Phys. Fluids 25, 409-410 (1982).

${ }^{17}$ C. C. Church, "The effects of an elastic solid surface layer on the radial pulsations of gas bubbles," J. Acoust. Soc. Am. 97, 1510-1521 (1995).

${ }^{18}$ A. Prosperetti, "Thermal effects and damping mechanisms in the forced radial oscillations of gas bubbles in liquids," J. Acoust. Soc. Am. 61,
17-27 (1977).

${ }^{19}$ R. E. Apfel and C. K. Holland, "Gauging the likelihood of cavitation from short-pulse, low-duty cycle diagnostic ultrasound," Ultrasound Med. Biol. 17, 179-185 (1991).

${ }^{20}$ B. E. Noltingk and E. A. Neppiras, "Cavitation produced by ultrasonics," Proc. Phys. Soc. London, Sect. B 63, 674-685 (1950).

${ }^{21}$ H. G. Flynn, "Cavitation dynamics. II. Free pulsations and models for cavitation bubbles,” J. Acoust. Soc. Am. 58, 1160-1170 (1975).

${ }^{22}$ H. G. Flynn and C. C. Church, "Transient pulsations of small gas bubbles in water," J. Acoust. Soc. Am. 84, 985-998 (1988).

${ }^{23}$ A. I. Eller, "Damping constants of pulsating bubbles," J. Acoust. Soc. Am. 47, 1469-1470 (1970).

${ }^{24}$ W. Lauterborn, "Numerical investigation of nonlinear oscillations of gas bubbles in liquids," J. Acoust. Soc. Am. 59, 283-293 (1976).

${ }^{25}$ C. K. Holland and R. E. Apfel, "An improved theory for the prediction of microcavitation thresholds," IEEE Trans. Ultrason. Ferroelectr. Freq. Control 36, 204-208 (1989).

${ }^{26}$ X. Yang, R. A. Roy, and R. G. Holt, "Bubble dynamics and size distributions during focused ultrasound insonation," J. Acoust. Soc. Am. 116, 3423-3431 (2004). 